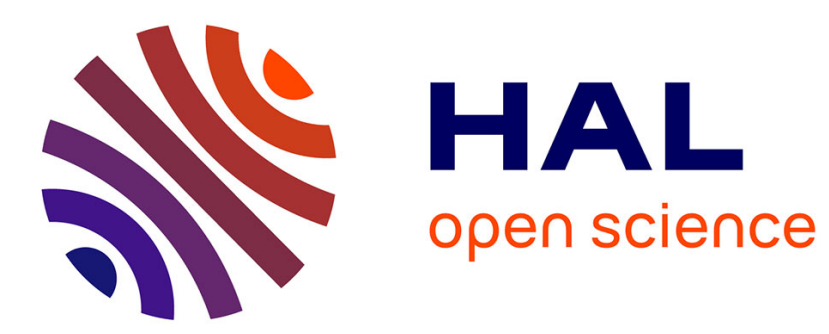

\title{
Etude d'une nouvelle utilisation des transistors de puissance dans les convertisseurs haute tension à fréquence élevée
}

\author{
Y. Cheron, H. Foch, J. Roux
}

\section{- To cite this version:}

Y. Cheron, H. Foch, J. Roux. Etude d'une nouvelle utilisation des transistors de puissance dans les convertisseurs haute tension à fréquence élevée. Revue de Physique Appliquée, 1981, 16 (6), pp.333342. 10.1051/rphysap:01981001606033300 . jpa-00244924

HAL Id: jpa-00244924

https://hal.science/jpa-00244924

Submitted on 1 Jan 1981

HAL is a multi-disciplinary open access archive for the deposit and dissemination of scientific research documents, whether they are published or not. The documents may come from teaching and research institutions in France or abroad, or from public or private research centers.
L'archive ouverte pluridisciplinaire HAL, est destinée au dépôt et à la diffusion de documents scientifiques de niveau recherche, publiés ou non, émanant des établissements d'enseignement et de recherche français ou étrangers, des laboratoires publics ou privés. 


\title{
Etude d'une nouvelle utilisation des transistors de puissance dans les convertisseurs haute tension à fréquence élevée
}

\author{
Y. Cheron, H. Foch et J. Roux \\ Institut National Polytechnique, Laboratoire d'Electrotechnique et d'Electronique Industrielle $\left({ }^{*}\right)$, \\ 2, rue Camichel, 31071 Toulouse Cedex, France
}

(Reçu le 3 février 1981, accepté le 4 mars 1981)

\begin{abstract}
Résumé. - Les auteurs définissent un nouveau composant : le thyristor dual qui peut être réalisé à partir de transistors de puissance. Après avoir indiqué les règles d'utilisation de ce composant, ils proposent une structure de convertisseur continu/continu, à étage intermédiaire haute fréquence, susceptible d'exploiter au mieux les propriétés du thyristor-dual. Une étude analytique de ce convertisseur permet d'en évaluer les caractéristiques et les limitations. Une représentation graphique permet de visualiser le mécanisme de la commutation. Une simulation et l'expérimentation d'un convertisseur de $2,5 \mathrm{~kW}$ alimenté en haute tension, permettent non seulement de confirmer les résultats de l'étude analytique, mais font apparaître les propriétés exceptionnelles de ce nouveau convertisseur à très haut rendement et puissance massique importante.
\end{abstract}

\begin{abstract}
The authors define a new component : the dual-thyristor that may be constructed using power transistors. After they have given the utilization rules of this component, they propose a structure of a DC/AC/DC converter with a high frequency link, able to take full advantage of the properties of the dual-thyristor. A theoretical analysis of this converter gives its characteristics and limitations. A graphic display shows the switching mechanism. Simulation and experimental results of a high voltage $2,5 \mathrm{~kW}$ converter not only confirm the results of the above mentioned theoretical analysis, but also show the very high efficiency and very high power to weight ratio of this new converter.
\end{abstract}

1. Introduction. - La recherche de performances élevées dans les convertisseurs statiques a suscité de nombreuses études tant dans les domaines de pointe (électronique spatiale) que dans les domaines industriels (haute tension).

Ces études mènent généralement à des structures fonctionnant en haute tension, pour améliorer le rendement, et en haute fréquence pour augmenter la puissance massique.

Dans le cas où un isolement galvanique est nécessaire, le transformateur de liaison bénéficie de la réduction de taille due à la haute fréquence. Ce même transformateur devient aussi indispensable dès qu'un rapport de tension très important doit être crée entre les tensions d'entrée et de sortie (chargeur de batteries ou générateur HT à partir du réseau industriel par exemple).

La haute fréquence et le rendement élevé sont cependant difficilement compatibles en raison des pertes par commutation des semi-conducteurs. Aussi de nombreux travaux ont porté sur des améliorations de la commutation dans ces convertisseurs.

L'utilisation de circuits accordés, fonctionnant au voisinage de leur fréquence de résonance, apporte

(*) ERA No 536. d'une part des commutations intéressantes et d'autre part une possibilité de réglage de puissance par variation du déphasage relatif courant-tension.

Cette voie, étudiée notamment pour les onduleurs moyenne fréquence de chauffage par induction [1] et plus récemment pour des convertisseurs continucontinu $[2,3,4]$ est bien connue pour les convertisseurs à thyristors.

Pour les faibles puissances, le remplacement des thyristors par des transistors de puissance a permis une nette amélioration des performances [5].

Cependant, les mécanismes de commutation de ces deux composants étant fondamentalement différents, l'optimisation de leur commutation ne peut les rendre interchangeables.

Les conditions de commutation optimale des transistors peuvent être déduites de celles des thyristors suivant une loi qui tient compte de leurs differences de fonctionnement.

Pour cela, les auteurs sont amenés à définir la notion de thyristor-dual, composant dont les propriétés sont duales de celles du thyristor et qui, réalisé avec des transistors de puissance, peut bénéficier d'une commutation à très faibles pertes. Ce composant bénéficie en outre d'une sûreté de fonctionnement très supérieure à celle que peuvent avoir le thyristor ou le transistor utilisé de manière traditionnelle. 
Les auteurs étudient alors un nouveau type de convertisseur continu/continu à étage intermédiaire haute fréquence utilisant des thyristors-duaux.

Pour la simplicité de l'exposé, le rapport de transformation du transformateur d'isolement est supposé unitaire et le transformateur négligé.

Une étude analytique, confirmée par une simulation, permet de prédéterminer les caractéristiques de ce convertisseur.

Une étude expérimentale sur une maquette de quelques kilowatts illustre la faisabilité et l'intérêt d'un tel convertisseur.

2. Le thyristor-dual. - Le transistor de puissance présentant la propriété de pouvoir être bloqué lorsqu'un courant positif le parcourt, comme le thyristor celle de pouvoir être rendu conducteur lorsqu'il a une tension positive à ses bornes, il paraît intéressant de définir les caractéristiques du composant électronique qui se présenterait comme le dual du thyristor.

Les caractéristiques et les propriétés du thyristor sont rappelées figure 1 et celles du thyristor-dual en sont déduites.

Ce composant dual n'existant pas naturellement, il est réalisé à l'aide d'un transistor de puissance muni

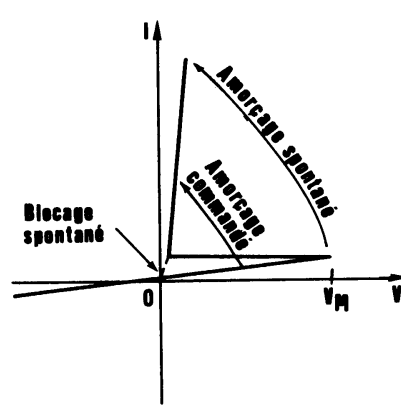

- Conditions d'amorçage $V \geqslant 0$ et ordre d'amorçage.

- Condition d'extinction $I \leqslant 0$.

$-I \leqslant I_{\mathrm{Max}}$.

- Protection contre $\mathrm{d} i / \mathrm{d} t$ à l'amorçage : inductance série.

- Amorçage spontané si $V=V_{\mathrm{M}}$.

[- Turn-on condition $V \geqslant 0+$ turn-on order.

- Turn-off condition $I \leqslant 0$.

$-I \leqslant I_{\text {Max }}$.

- Protection against $\mathrm{d} i / \mathrm{d} t \mathrm{dur}-$ ing turn-on : series inductance.

- Spontaneous turn-on if $V=V_{\mathbf{M}}$.]

Fig. 1. - Caractéristiques et propriétés du thyristor et du thyristor dual.

[Thyristor and dual-thyristor properties.] de circuits spécifiques de commande et d'une diode tête-bêche.

L'amorçage qui s'effectue au zéro de tension et la commande à l'ouverture réduisent le circuit d'aide à la commutation à un simple condensateur en parallèle sur le transistor.

La figure 2 montre un exemple de réalisation de la commande de base du transistor de puissance. $T_{p}$ est le transistor principal, $\mathrm{D}$ la diode de récupération et $C$ le condensateur d'aide à la commutation.

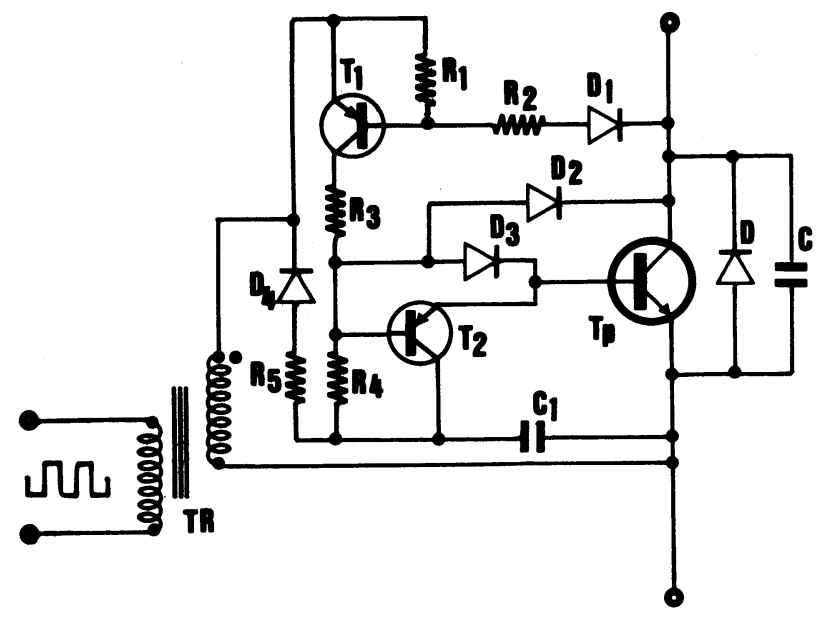

Fig. 2. - Exemple de réalisation de thyristor dual.

[Example of dual-thyristor.]

$\mathrm{T}_{1}$ est le transistor d'alimentation directe de base à travers la résistance $R_{3}$ et le circuit autorégulant $\mathrm{D}_{2}$, $D_{3}$. Ce circuit permet d'entretenir une saturation correcte de $T_{p}$.

$T_{1}$ réalise aussi la logique de commande car il ne conduit que si TR fournit une alimentation positive sur son émetteur ET si $R_{2} D_{1}$ est rendu conducteur par l'annulation de la tension aux bornes de $T_{p}$.

$\mathrm{T}_{2}$ est le transistor d'extinction et le réseau $\mathrm{D}_{4} R_{5}$ permet de garder $C_{1}$ chargé afin de permettre à la fonction disjoncteur de réaliser si nécessaire une polarisation négative de base.

On peut noter qu'un seul transformateur TR à deux secondaires permet la commande d'un bras complet d'onduleur.

Le cycle $I_{\mathrm{C}}\left(V_{\mathrm{CE}}\right)$ du transistor est un cycle à très faibles pertes et une polarisation négative de la base permet au transistor de supporter les plus hautes tensions (tenue en tension $V_{\text {CEX }}$ des transistors). Ces deux propriétés destinent naturellement cet interrupteur à être utilisé dans les convertisseurs fonctionnant à fréquence et tension élevées.

D'autre part, en se présentant comme le dual du thyristor, il sera utilisé dans les meilleures conditions dans les structures duales de celles où le thyristor se bloque naturellement.

Les auteurs proposent d'étudier un convertisseur continu-continu à étage intermédiaire moyenne fréquence. D'après la remarque précédente, la structure 
de l'onduleur moyenne fréquence utilisant ces thyristors duaux sera celle d'un onduleur de tension (ou onduleur série) (Fig. 3).

Cette structure utilisant des thyristors a déjà été largement étudiée. L'utilisation du transistor de puissance a permis d'atteindre des fréquences élevées et des rendements exceptionnels. Cependant, une commande de ces transistors sensiblement équivalente à celle des thyristors ne modifie pas les propriétés de la structure.

L'utilisation des thyristors-duaux permet au contraire d'envisager des modifications des propriétés de la structure tout en gardant les performances liées au transistor de puissance.

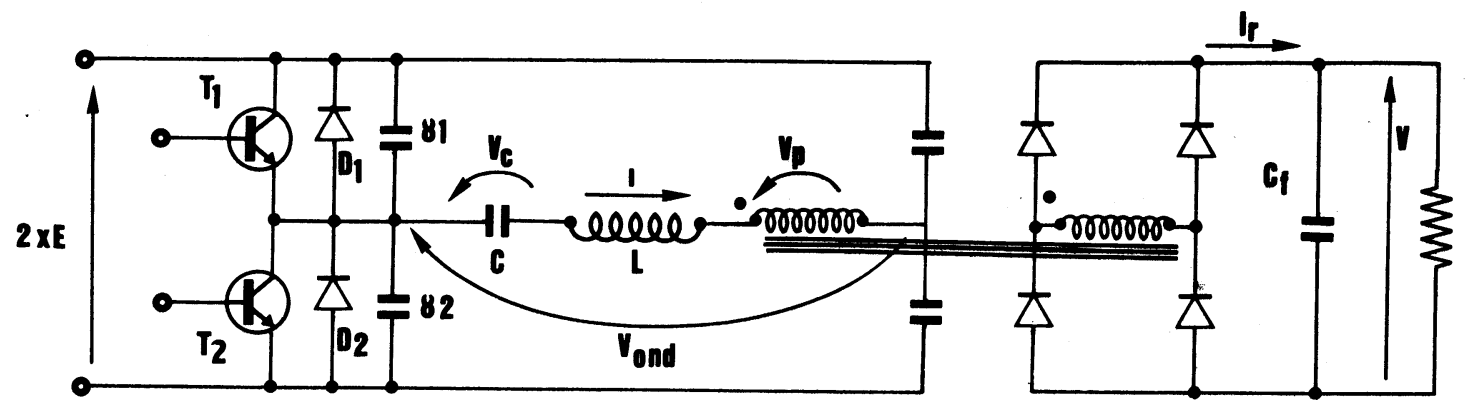

Fig. 3. - Structure du convertisseur étudié.

[Structure of the studied converter.]

3. Etude qualitative. - Cette étude a été menée dans le cadre d'une simulation analogique globale par le procédé Cassis [9]. Cette méthode de simulation présente, grâce à un câblage topologiquement conforme, les avantages conjoints d'une simulation analogique et d'une maquette.

Les composants passifs tels que résistances, inductances, capacités sont simulés analogiquement par des équations différentielles et sont reliés aux interrupteurs statiques par des interfaces permettant de rendre indépendant chaque composant.

Pour effectuer cette simulation qualitative, le transformateur est supposé avoir un rapport de transformation unitaire et un courant magnétisant nul. Cette hypothèse permet de s'affranchir du transformateur dans toute l'étude présentée.

La figure 4 représente les formes d'ondes caractéristiques de ce convertisseur.

Cette simulation permet aussi de justifier les hypothèses simplificatrices supplémentaires faites pour l'étude analytique en régime permanent :

- les composants sont parfaits,

- les commutations des semi-conducteurs sont instantanées,

- la charge du redresseur est assimilée à une source de tension parfaite $V$,

- le point milieu capacitif est supposé idéal.

4. Etude analytique. -4.1 Notations.

$$
\begin{aligned}
\gamma_{1} & =\gamma_{2}=\gamma / 2 \\
1 / \mathcal{C} & =1 / C+1 / \gamma \\
C & =\alpha \gamma \\
k & =(\alpha+1)^{1 / 2} . \\
\omega_{\mathrm{r}} & =(L C)^{-1 / 2}=2 \pi f_{\mathrm{r}} \\
\omega_{\mathrm{r}}^{*} & =(L C)^{-1 / 2}
\end{aligned}
$$

$f$ : fréquence de fonctionnement du convertisseur, $\theta:$ angle de conduction d'un transistor,

$\theta_{c}:$ angle de commutation,

$\theta_{\mathrm{D}}:$ angle de conduction d'une diode.

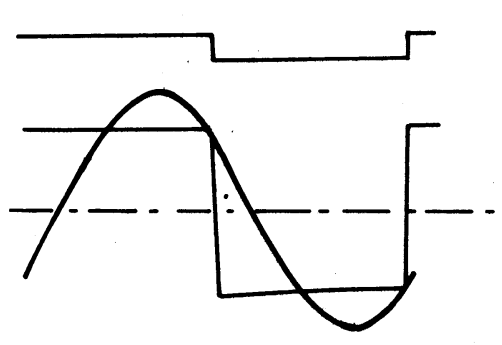

- Commande de $T_{1}$. [Base drive of $T_{1}$.]

- Tension aux bornes de l'onduleur : $V_{\text {ond }}$.

[Output voltage of the inverter : $V_{\text {ond }}$.]

- Courant $I$. [Current I.]
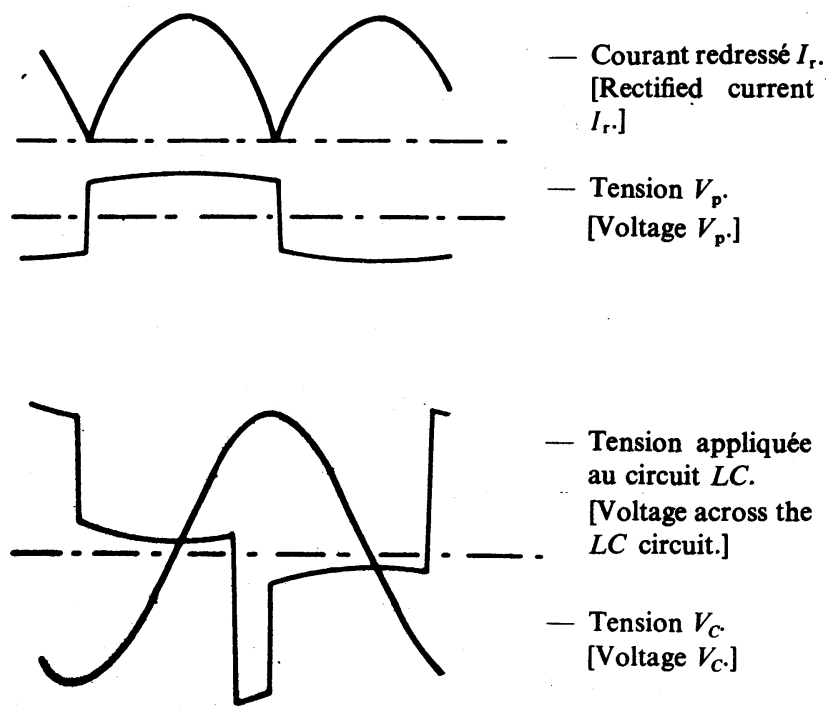

Fig. 4. - Formes d'ondes caractéristiques de ce convertisseur. [Characteristic waveforms of the converter.] 


\subsection{UNITÉS RÉDUITES.}

$$
\begin{gathered}
x=v_{c} / E ; \quad x^{*}=v_{\mathrm{e}} / E \\
y=(i / E) \sqrt{L / C} ; \quad y^{*}=(i / E) \sqrt{L / C} \\
q=V / E
\end{gathered}
$$

$x_{\mathrm{M}}$ : Tension maximale aux bornes du condensateur $C$.

$y_{M}$ : Courant crête dans les transistors.

$y_{\text {moy }}$ : Courant dans la charge.

En régime permanent, l'étude du fonctionnement de ce convertisseur est réduite à l'étude de trois séquences élémentaires dont les circuits simplifiés sont représentés figure 5 :

- conduction du transistor $T_{1}(a)$,

- commutation du transistor $\mathrm{T}_{1}$ sur la diode $\mathrm{D}_{2}(b)$,

- conduction de la diode $\mathrm{D}_{2}(c)$.

(a)

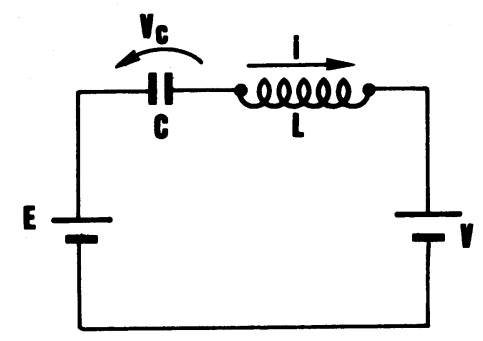

(b)

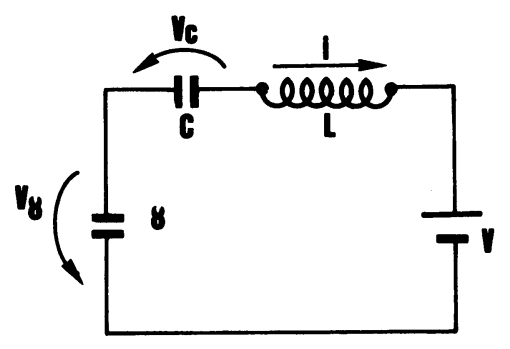

(c)

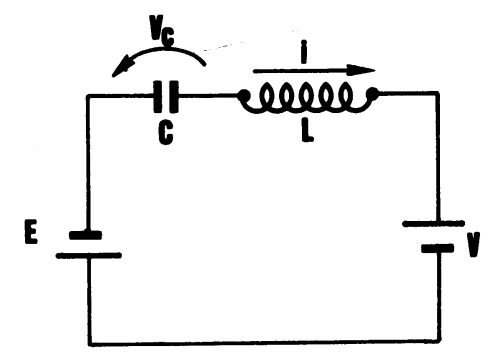

Fig. 5.

Chacun de ces circuits étant composé d'un circuit oscillant de coefficient de qualité supposé infini excité par une source de tension, il paraît très intéressant de représenter l'évolution des grandeurs caractéristiques de ce circuit oscillant dans le plan de phase $\left(v_{c}, i \sqrt{L / C}\right)$.

On peut ajouter encore plus de généralité à cette représentation en utilisant les grandeurs réduites $x, y$ et $q$, la demi-tension d'alimentation valant alors +1 .

Ne pouvant représenter dans le plan de phase que des grandeurs alternatives sinusoïdales de même pulsation, le fonctionnement de ce convertisseur sera représenté dans deux plans de phase différents puisque les grandeurs alternatives évoluent à la pulsation $\omega_{\mathrm{r}}$ pendant la conduction des semi-conducteurs $T_{1}$ et $D_{2}$ et à la pulsation $\omega_{\mathrm{r}}^{*}$ pendant les comınutations.

Les règles de passage entre ces deux plans de phase sont directement déduites des expressions de $x^{*}$ et $y^{*}$ avant la commutation :

$$
\left\{\begin{array}{l}
x_{0}^{*}=x_{0}-1 \\
y_{0}^{*}=k y_{0}
\end{array}\right.
$$

et après la commutation :

$$
\left\{\begin{array}{l}
x_{\mathrm{f}}^{*}=x_{\mathrm{f}}+1 \\
y_{\mathrm{f}}^{*}=k y_{\mathrm{f}}
\end{array}\right.
$$

Un thyristor-dual ne peut s'amorcer que si la commande le lui permet ET si la tension à ses bornes s'est annulée. La commutation ne sera donc parfaitement assurée que si les deux conditions suivantes sont vérifiées :

$$
\left\{\begin{array}{l}
x_{\mathrm{f}}^{*}=x_{0}+2+\frac{2}{\alpha} \\
y_{\mathrm{f}}^{*} \geqslant 0 .
\end{array}\right.
$$

Ces deux conditions traduisent de façon analytique le fait que le condensateur $\gamma$ doit avoir inversé sa polarité avant que le courant ne s'annule.

En superposant ces deux plans de phase, on passe donc aisément de l'un à l'autre au moyen d'une homothétie suivant l'axe des $y$ et d'une translation suivant l'axe des $x$.

La représentation du fonctionnement du convertisseur sur une demi-période est donnée figure 6.

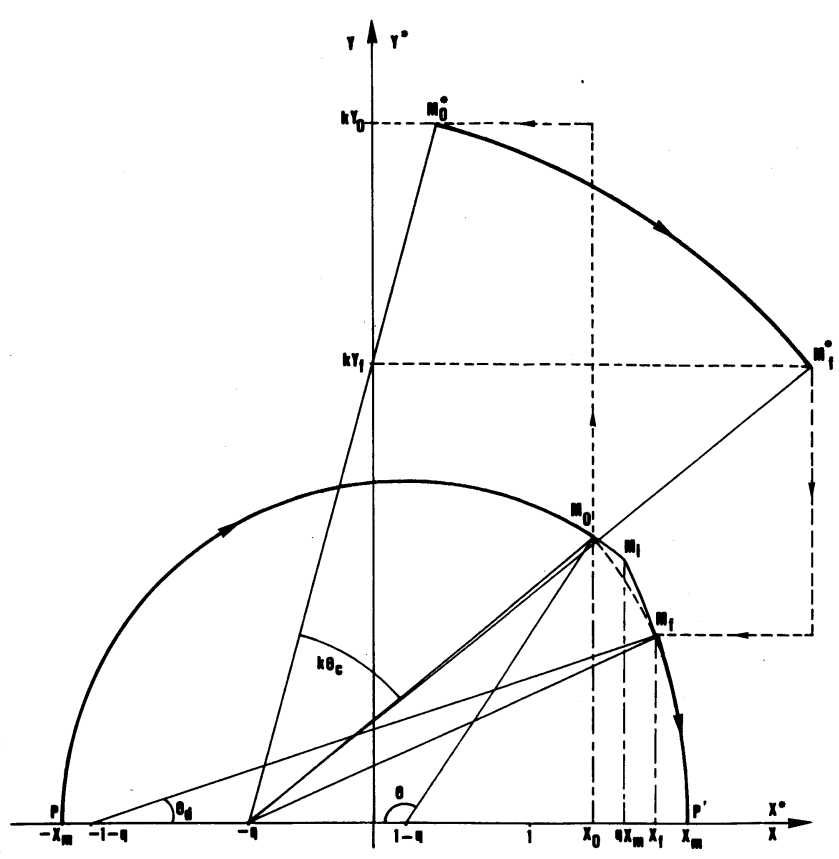

Fig. 6. - Représentation dans le plan de phase.

[Display in the phase plane.] 
La conduction du transistor $T_{1}$ est représentée par l'arc de cercle $\widehat{\mathrm{PM}}_{0}$, la conduction de la diode $\mathrm{D}_{2}$ par l'arc de cercle $\widehat{\mathbf{M}_{\mathbf{f}} \mathbf{P}^{\prime}}$ et la commutation par l'arc de cercle $\overline{\mathrm{M}_{0}^{*} \mathrm{M}_{1}^{*}}$.

Le point $\mathbf{M}_{\mathrm{i}}$ représente la commutation quand il n'y a pas de condensateur d'aide à la commutation, toutes choses étant égales par ailleurs. On peut noter l'abscisse particulière de ce point $M_{i}$ et la symétrie des abscisses des points $\mathbf{M}_{0}$ et $\mathbf{M}_{\mathrm{f}}$ par rapport à cette dernière.

4.3 CaRaCtÉRISTIQUes en CHARge. - A partir de cette représentation, l'écriture des expressions des différentes grandeurs de ce convertisseur est immédiate si l'on choisit $\theta$ et $\alpha$ comme paramètres et $q$ comme variable.

Le choix de $\theta$ comme paramètre est justifié par le fait que les transistors sont commandés à l'ouverture. De façon analogue, dans les convertisseurs à thy- ristors, le choix de l'angle de conduction des diodes est justifié par le fait que les thyristors sont commandés à la fermeture.

Cependant, à moins de réaliser un autopilotage du convertisseur, la commande du système à $\theta$ constant est difficile à réaliser. Pratiquement, une commande à fréquence constante est directement accessible.

D'autre part, si la charge est passive, $q$ n'est plus une variable pour le système. $\theta$ (ou $f$ ) devient la variable de réglage du transfert d'énergie, $\alpha$ restant le seul paramètre.

A l'aide des relations suivantes, les caractéristiques à fréquence constante, paramétrées en $f / f_{\mathrm{r}}$, peuvent être tracées dans le plan $q\left(y_{\text {moy }}\right)$ (Fig. 7a).

$$
\begin{aligned}
x_{\mathrm{M}} & =\frac{1 / \alpha+(1-q)(1-\cos \theta)}{q+\cos \theta} \\
x_{0} & =q x_{\mathrm{M}}-1 / \alpha \\
x_{\mathrm{f}} & =q x_{\mathrm{M}}+1 / \alpha
\end{aligned}
$$

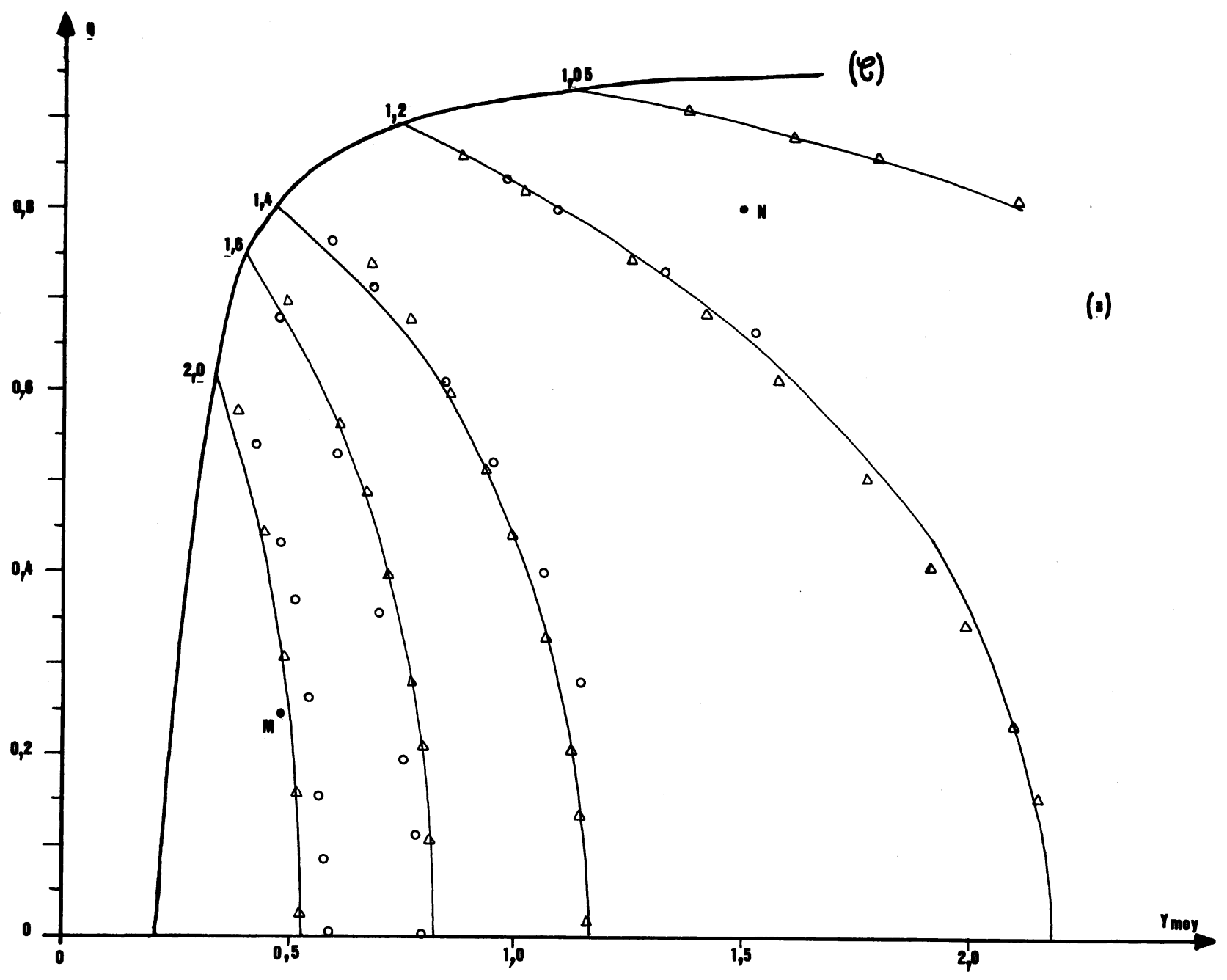

Fig. 7. - Caractéristiques à fréquence constante et à courant crête constant dans transistors $(\alpha=10)$. C Courbes théoriques. $\Delta$ Points obtenus par simulation. O Points expérimentaux. (C) Courbe critique de commutation. a) Caractéristiques à fréquence constante, paramétrées en $f\left(f_{\mathrm{r}}, b\right)$ Caractéristiques à courant crête constant dans les transistors, paramétrées en $y_{\mathrm{M}}$.

[Characteristics at a constant frequency and at a constant peak current in the transistors $(\alpha=10)$. - Theoretical curves. $\Delta$ Simulation results. $O$ Experimental results. (C) Critical commutation line. $a$ ) Characteristics at a constant frequency, with $f\left(f_{\mathrm{r}}\right.$ as a parameter. $b$ ) $C$ Characteristics at a constant peak current, with $y_{M}$ as a parameter.] 


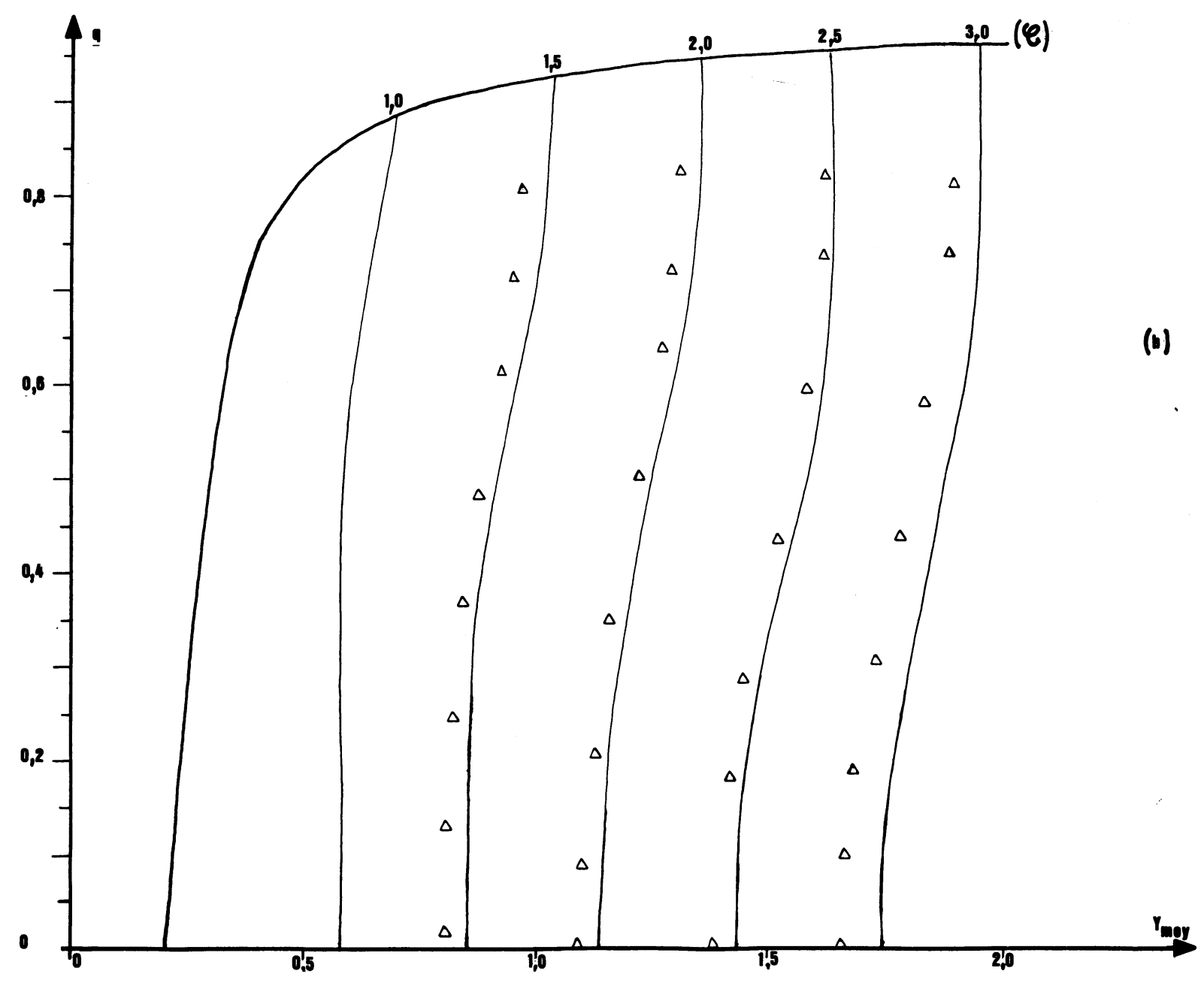

$$
\begin{aligned}
\theta_{\mathrm{c}} & =\frac{1}{k}\left(\operatorname{arctg} \frac{k y_{0}}{x_{0}+q-1}-\operatorname{arctg} \frac{k y_{\mathrm{f}}}{x_{\mathrm{f}}+q+1}\right) \\
\theta_{\mathrm{D}} & =\operatorname{arctg} \frac{y_{\mathrm{f}}}{x_{\mathrm{f}}+q+1} \\
f / f_{\mathrm{r}} & =\pi /\left(\theta+\theta_{\mathrm{c}}+\theta_{\mathrm{D}}\right) \\
y_{\text {moy }} & =\frac{f}{f_{\mathrm{r}}} \frac{2 x_{\mathrm{M}}}{\pi} .
\end{aligned}
$$

Pour le dimensionnement des semi-conducteurs, il est indispensable de connaître' les valeurs crêtes du courant dans les transistors. D'après la figure 6 , on obtient :

$$
\begin{array}{lll}
\text { si } & \theta>\pi / 2 & y_{M}=x_{M}+1-q \\
\text { si } & \theta<\pi / 2 & y_{M}=\left(x_{M}+1-q\right) \sin \theta
\end{array}
$$

et les caractéristiques à $y_{\mathrm{M}}$ constant peuvent être tracées dans le plan $q\left(y_{\text {moy }}\right)$ (Fig. $\left.7 b\right)$.

4.4 EXemple D'application. - Afin de mettre en évidence l'intérêt pratique d'un tel dispositif et de vérifier la méthode d'étude analytique présentée, un convertisseur fonctionnant sur le principe énoncé, travaillant à fréquence inaudible, alimenté par un réseau triphasé $380 \mathrm{~V}$ redressé et capable de délivrer une puissance de $2,5 \mathrm{~kW}$ sous $200 \mathrm{~V}$ a été réalisé. Pour rester en accord avec l'étude théorique, le transformateur a été supprimé.

Le cahier des charges impose $E \# 250 \mathrm{~V}$ et $V \# 200 \mathrm{~V}$, c'est-à-dire $q=0,8$.

Le convertisseur fonctionne toujours à une fréquence supérieure à la fréquence de résonance. Afin de rester dans le domaine des fréquences ultrasoniques, la fréquence de résonance $f_{\mathrm{r}}$ est fixée à $23 \mathrm{kHz}$.

Le point de fonctionnement doit être choisi suffisamment éloigné de la courbe critique (C) de commutation pour être stable (Fig. 7). En effet, une variation de la tension d'alimentation se traduit par une variation de $q$ et si le point de fonctionnement est choisi près de la courbe critique, le défaut de commutation et l'arrêt de l'onduleur sont à craindre. Cette remarque amène à choisir $y_{\text {moy }}=1,5$.

Ce point de fonctionnement nominal est repéré par $\mathrm{N}$ sur la figure $7 a$. Ce point appartient à une caractéristique telle que $f / f_{\mathrm{r}}$ soit voisin de 1,1 , c'est-àdire $f$ voisine de $25 \mathrm{kHz}$.

La puissance délivrée par l'onduleur s'écrit :

soit encore :

$$
P=V I_{\text {moy }}
$$

$$
P=q y_{\mathrm{moy}} E^{2} \sqrt{C / L} \text {. }
$$


Des expressions de la puissance et de la fréquence de résonance, on déduit les valeurs de $L$ et de $C$ :

$$
\begin{aligned}
& L=217 \mu \mathrm{H} \\
& C=0,22 \mu \mathrm{F} .
\end{aligned}
$$

Pour le point de fonctionnement choisi et d'après les caractéristiques de la figure $7 b$, le courant maximal dans les transistors vaut :

soit

$$
I_{\mathrm{M}}=2,3 E \sqrt{C / L}
$$

$$
I_{\mathrm{M}} \# 18 \mathrm{~A} \text {. }
$$

A la vue des contraintes en courant et tension, les semi-conducteurs de puissance peuvent être choisis comme suit :

- les transistors $T_{1}$ et $T_{2}$ sont constitués de 2 BUX 48 en parallèle attaqués en Darlington par un troisième,

- les diodes $D_{1}$ et $D_{2}$ sont deux diodes BYX 66800 .

L'utilisation de BUX 48

$$
\left(V_{\mathrm{CEO}}=400 \mathrm{~V}, V_{\mathrm{CEX}}=850 \mathrm{~V}\right)
$$

permet d'avoir une marge de sécurité confortable sur les tensions dans un fonctionnement en $V_{\text {CEX }}$.

Afin de réduire les pertes à l'ouverture des transistors et d'assurer une parfaite tenue en tension $V_{\text {CEX }}$, la tension $V_{\mathrm{CE}}$ lorsque le transistor a fini de se bloquer doit être inférieure à $V_{\text {CEO }}$. En conséquence des condensateurs d'aide à la commutation de $10 \mathrm{nF}$ ont été choisis et on en déduit :

$$
\alpha=10 \text {. }
$$

Les condensateurs du point milieu et de filtrage sont choisis de façon à vérifier les hypothèses de l'étude analytique.

4.5 Résultats expérimentaux. - A l'aide de ce montage et d'une simulation analogique quantitative, des caractéristiques à fréquence constante et à courant crête constant ont été relevées (Fig. 7). Il faut remarquer que ces caractéristiques s'insèrent dans un réseau et que les positions relatives des caractéristiques théoriques et expérimentales dépendent essentiellement de la précision de connaissance des paramètres du montage.

Les photos de la figure 8 montrent les formes d'ondes expérimentales pour le point de fonctionnement nominal, $\mathrm{N}$, à $2,5 \mathrm{~kW}$. Sans modifier la résistance de charge mais avec une fréquence voisine de $50 \mathrm{kHz}$, le point de fonctionnement figuré par $\mathrm{M}$ sur la figure $7 a$ a été obtenu. Les formes d'ondes caractéristiques de ce point sont représentées à la figure 9. La puissance délivrée par le convertisseur dans ces conditions est de l'ordre du dixième de la puissance nominale.

4.6 INFLUENCE DES CONDENSATEURS $\gamma_{1}$ ET $\gamma_{2}$. Le principe de commutation des transistors impose un condensateur auxiliaire pour limiter les gradients de tension à l'ouverture.
Dans les conditions de fonctionnement définies précédemment, la puissance dissipée du fait de la commutation des transistors est seulement de l'ordre de $1 \mathrm{~W}$ alors que la puissance dissipée par conduction est de l'ordre de $30 \mathrm{~W}$. La comparaison de ces deux puissances illustre certes l'efficacité d'un condensateur d'aide à l'ouverture mais montre surtout que ce condensateur est dimensionné pour le fonctionnement en $V_{\text {CEX }}$.

En outre, ce condensateur implique une frontière de commutation critique (C) au-delà de laquelle l'onduleur s'arrête (Fig. 7). Dans la zone où la commutation s'effectue normalement, un examen du transfert énergétique au sein de la charge et du circuit oscillant pendant la commutation transistor-diode permet d'évaluer la pénalisation qu'entraîne la présence de ces condensateurs auxiliaires.

D'après la relation (3), la commutation s'effectue normalement si :

$$
\alpha>\frac{1}{x_{M}(1-q)} .
$$

Ceci signifie aussi qu'il existe une valeur maximale de l'angle de conduction du transistor pour chaque valeur de la tension de charge $(q)$ et de la surtension aux bornes de $C\left(x_{\mathrm{M}}\right)$, pour laquelle les diodes de récupération ne conduisent plus.

L'énergie transmise à la charge pendant la commutation s'écrit :

$$
\mathcal{E}=q C E^{2}\left(x_{\mathrm{f}}-x_{0}\right) .
$$

Dans la mesure où les points $M_{0}$ et $M_{f}$ (Fig. 6) appartiennent à deux arcs de cercle qui ne dépendent que de $q$ et de $x_{M}$ et où la commutation s'effectue normalement, l'énergie transmise à la charge est indépendante de $\alpha$.

Globalement, pendant la commutation, il y a donc transfert d'énergie de l'inductance vers la charge et le condensateur $C$, les condensateurs $\gamma_{1}$ et $\gamma_{2}$ n'intervenant pas dans ce transfert. Ceci signifie aussi que si l'on court-circuitait la sortie de l'onduleur jusqu'à ce que le condensateur $C$ ait subi un accroissement de tension $2 E / \alpha$, le bilan énergétique dans la charge et le circuit oscillant serait le même. Cela se traduit dans le plan de phase (Fig. 6) par le fait que $M_{0}$ et $M_{f}$ appartiennent à un même arc de cercle centré sur le point de coordonnées :

$$
x=-q ; \quad y=0 .
$$

Il apparaît donc nécessaire de faire un compromis dans le choix des condensateurs auxiliaires $\gamma_{1}$ et $\gamma_{2}$ entre la tenue en tension $V_{\text {CEX }}$ des transistors et les limites de variation de la charge, étant bien entendu que plus les condensateurs de commutation sont petits, plus la limite de commutation est repoussée.

5. Conclusion. - En valeurs instantanées, le convertisseur se présente comme une source de tension (condensateur de filtrage à la sortie). 


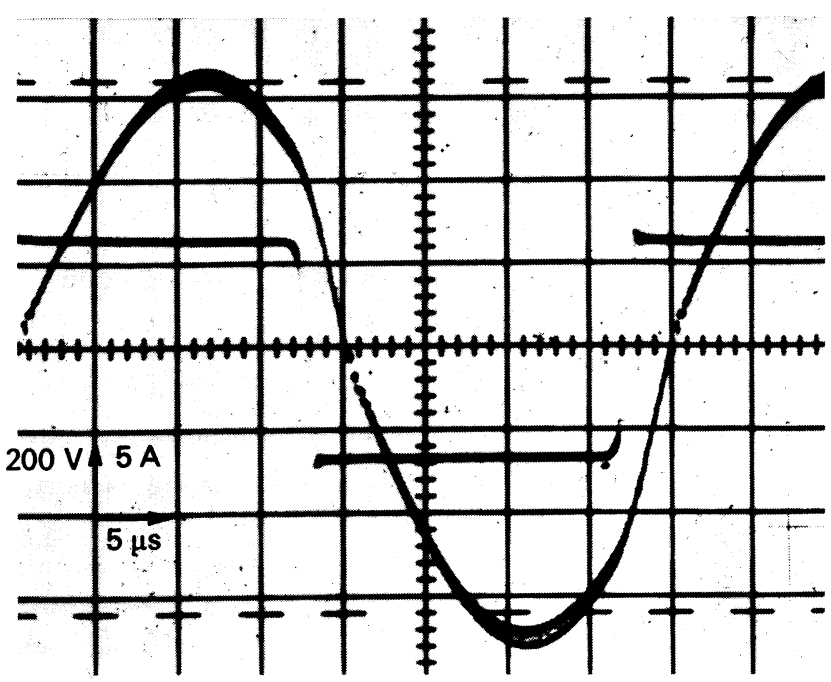

a)

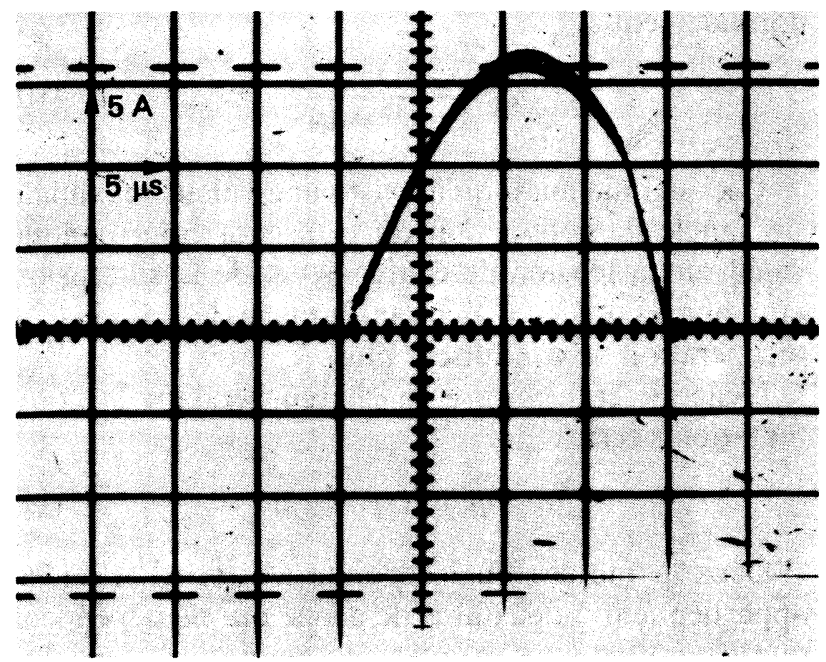

c)

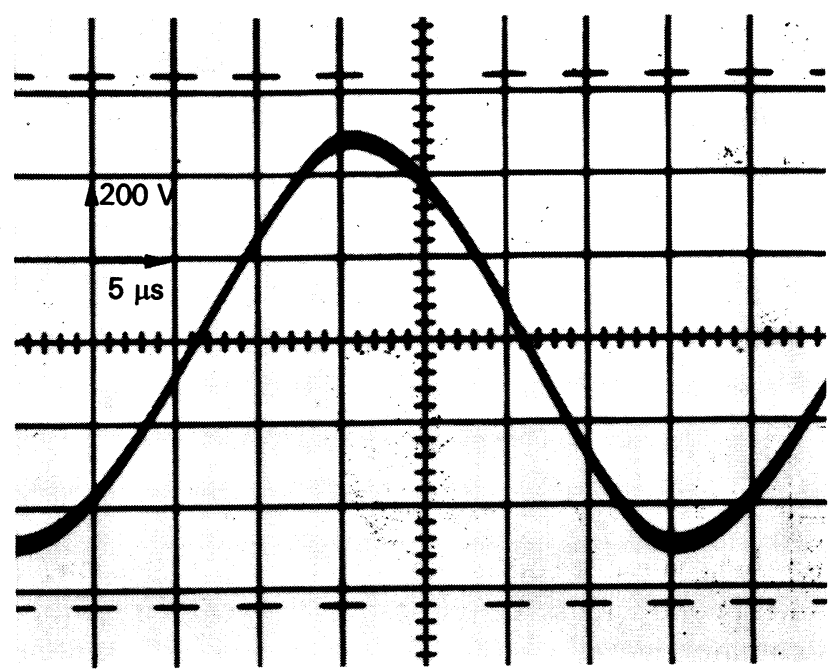

b)

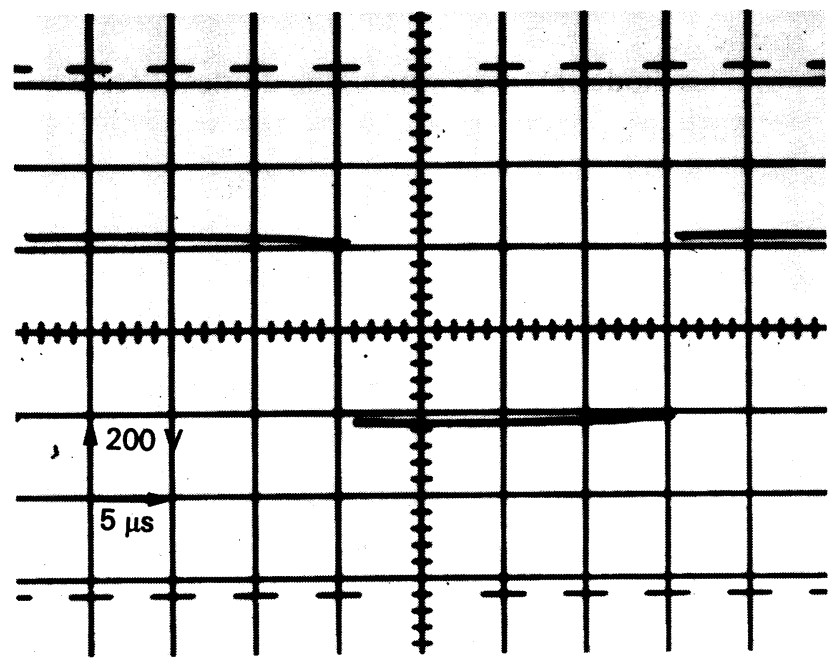

d)

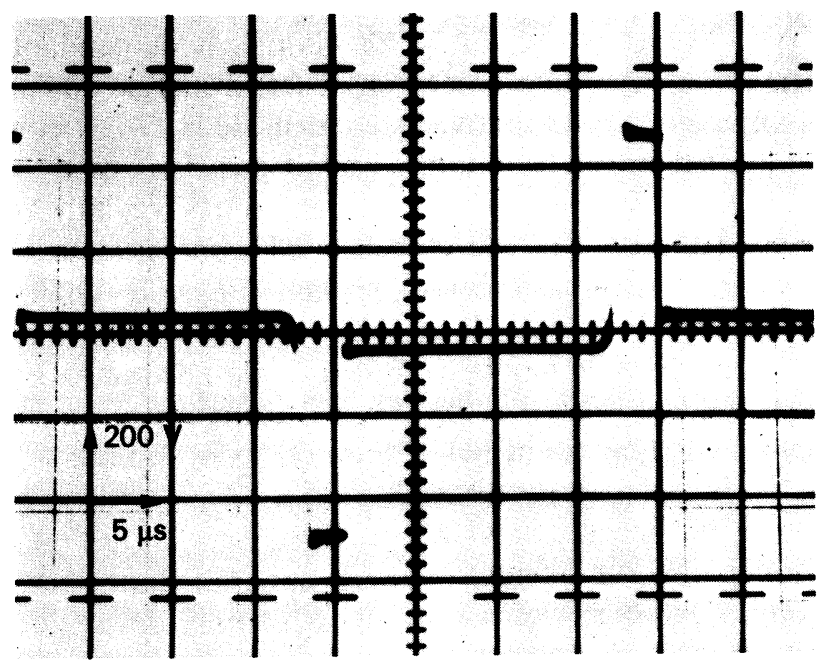

e)

Fig. 8. - Formes d'onde expérimentales pour le fonctionnement nominal $F=25 \mathrm{kHz}$. $a$ : Tension délivrée par l'onduleur $V_{\text {ond }}$ et courant dans le circuit oscillant $I . b:$ Tension aux bornes du condensateur $V_{c} . c:$ Courant dans une diode du pont redresseur. $d:$ Tension appliquée au pont redresseur. $e$ : Tension appliquée au circuit oscillant.

[Experimental waveforms for full power operating. $F=25 \mathrm{kHz} . a:$ Output voltage of the inverter and current in the $L C$ circuit. $b:$ Voltage across the capacitor: $V_{c} \cdot c$ : Current in a diode of the rectifier bridge. $d:$ Input voltage of the rectifier bridge. $e:$ Voltage across the $L C$ circuit.] 


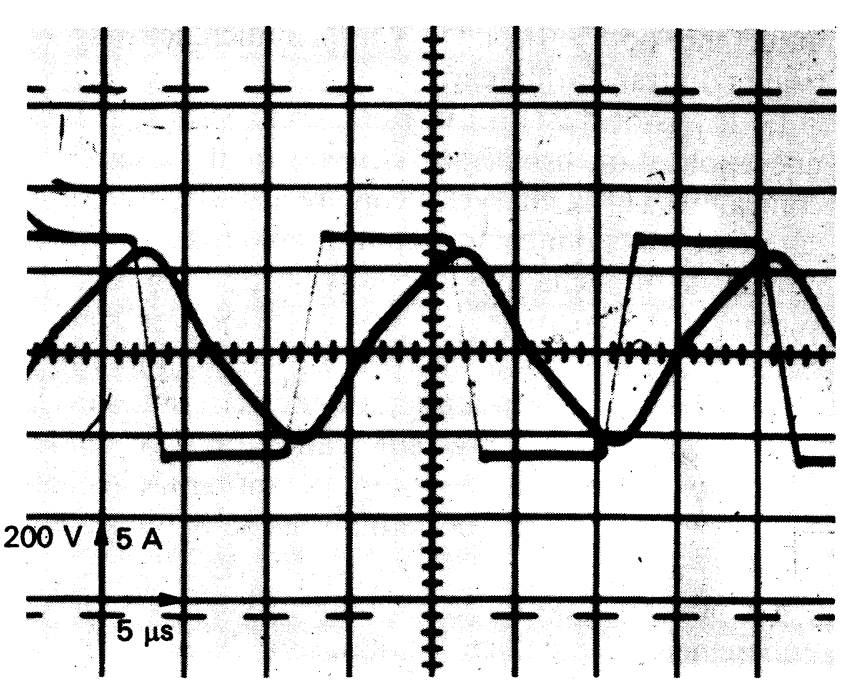

a)

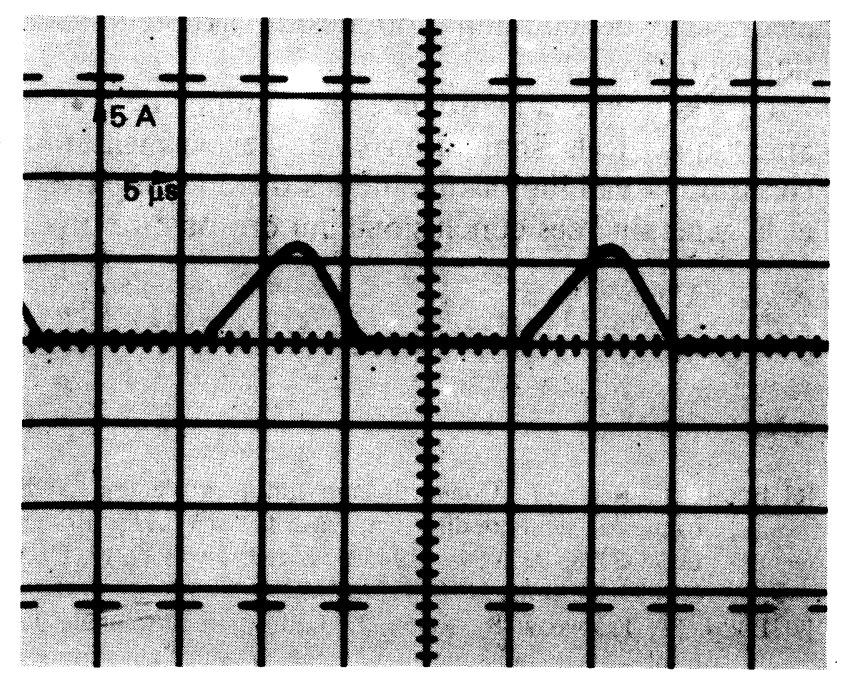

c)

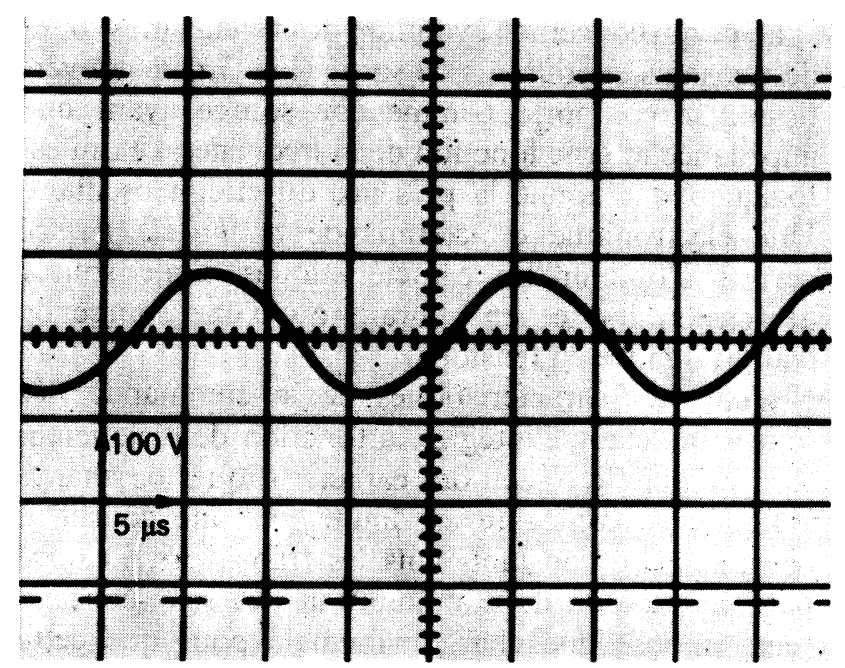

b)

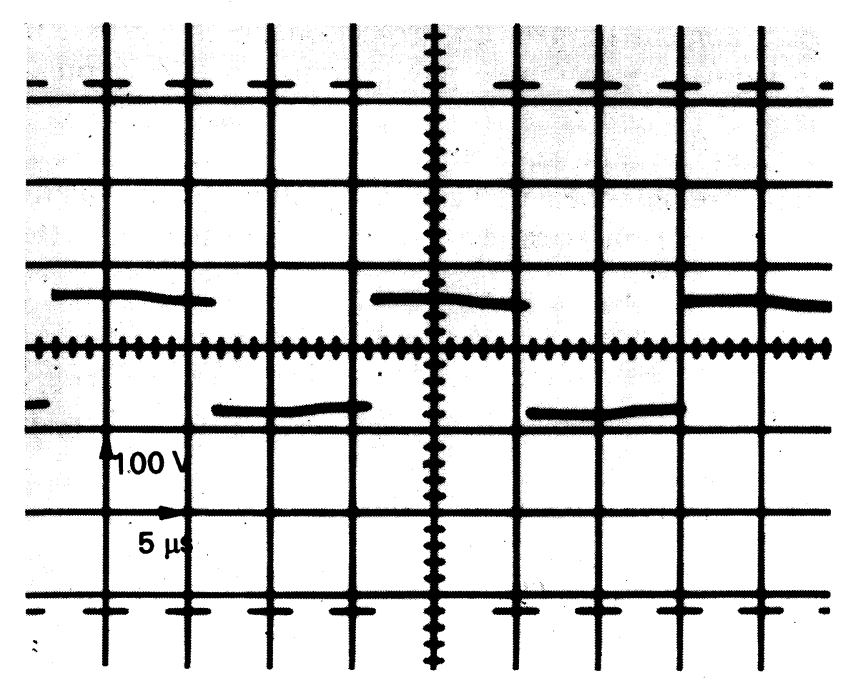

d)

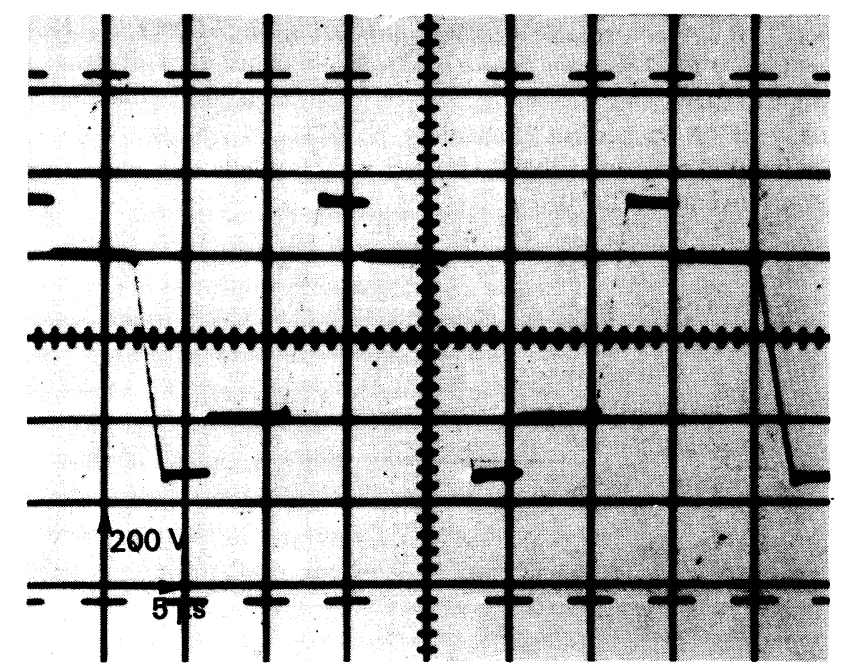

e)

Fig. 9. - Formes d'onde expérimentales pour le fonctionnement à puissance réduite $F=50 \mathrm{kHz}$. $a:$ Tension délivrée par l'onduleur $V_{\text {ond }}$ et courant dans le circuit oscillant $I . b:$ Tension aux bornes du condensateur $V_{c} . c:$ Courant dans une diode du pont redresseur. $d$ : Tension appliquée au pont redresseur. $e$ : Tension appliquée au circuit oscillant.

[Experimental waveforms for reduced power operation. $F=50 \mathrm{kHz} . a:$ Output voltage of the inverter and current in the $L C$ circuit. $b:$ Voltage across the capacitor : $V_{C} \cdot c:$ Current in a diode of the rectifier bridge. $e:$ Input voltage of the rectifier bridge. $e:$ Voltage across the $L C$ circuit.] 
En ce qui concerne l'évolution des valeurs moyennes des tensions et courants de sortie (Fig. 7a), le convertisseur se comporte comme une source ayant une impédance interne fonction de la fréquence, ce qui est logique étant donné la présence du circuit oscillant. Une électronique de commande en boucle fermée permet d'obtenir les caractéristiques d'une source de tension limitée en courant ou d'une source de courant limitée en tension.

L'une des caractéristiques de la commande des transistors étant d'intégrer la fonction de disjoncteur électronique, le réseau des caractéristiques de charge à fréquence constante est limité par une courbe à courant crête constant dans les transistors. D'autre part, le procédé de commutation des semi-conducteurs impose une charge minimale pour que cette commutation s'effectue normalement. La partie du plan où se déplace le point de fonctionnement est donc parfaitement limitée. La protection du convertisseur se trouve ainsi assurée par la limitation de la puissance.

Dans la pratique, il faut noter que la marche à vide peut être obtenue en plaçant une inductance de valeur convenable en parallèle sur le transformateur, cette inductance pouvant d'ailleurs être l'inductance magnétisante du transformateur.

Le thyristor-dual trouve donc dans cette structure une application privilégiée et, bien qu'il impose des contraintes sur la charge, il confère à la structure une sûreté de fonctionnement remarquable (pas de courtcircuit de branche possible, disjonction en cas de surcharge accidentelle) et un très bon rendement grâce à la diminution des pertes par commutation. D'autre part, en commutant au zéro du courant dans le circuit oscillant, le redresseur n'affecte pas ce rendement car, les gradients de courant étant naturellement faibles, les pertes par commutation sont quasiment nulles (Figs. 8c, 9c).

L'étude analytique de ce convertisseur est particulièrement allégée par l'utilisation de la méthode du plan de phase. Ce dernier permet de donner une image fidèle du fonctionnement et notamment du mécanisme de commutation des interrupteurs statiques utilisés.

La très bonne concordance des résultats de l'étude analytique, de la simulation et de l'expérimentation confirme la validité des méthodes d'étude employées et la généralité des conclusions qui ont pu être tirées.

\section{Bibliographie}

[1] Brichant, F., L'ondistor (Edition Dunod) 1970.

[2] Schwarz, F. C., An improved method of resonant current pulse modulation for power converters, IEEE Trans. IECI 23 mai (1976).

[3] Schwarz, F. C. and KlaAssens, J. B., A controlable secondary multikilowatt DC current source with constant maximum power factor in its three-phase supply line, IEEE Trans. IECI 23 May (1976).

[4] Schwarz, F. C. and KlaAssens, J. B., A controlable $45 \mathrm{~kW}$ current source for DC machines IEEE Trans. on Industry Applications IA-15 July/August (1979).

[5] Schwarz, F. C. and KlaAssens, J. B., A 95 percent efficient $1 \mathrm{~kW}$ DC converter with an internal frequency of $50 \mathrm{kHz}$, IEEE Trans. IECI 25, No 4, November (1978).
[6] Foch, H., Roux, J., Convertisseurs statiques d'énergie électrique à semi-conducteurs, Brevets $A N V A R$, France no 7832428, RFA No P29452457, GB. No 7939217, USA No 093106, Italie No 83487A/79.

[7] Foch, H., Trannoy, B., Roux, J., Utilisation rationnelle des transistors de puissance haute tension pour l'alimentation d'une machine asynchrone à partir du réseau $380 \mathrm{~V}$ $50 \mathrm{~Hz}$, AIM Liège, novembre (1978).

[8] Foch, H., Marty, P., Roux, J., Use of Duality Rules in the conception of transitorised converters, Power Conversion'80 Munich, 3-5 septembre (1980) 4B3, 1 à 11 .

[9] Metz, M., Méthode d'étude des convertisseurs statiques par simulation analogique, Modèles de semi-conducteurs, Simulation à câblage topologiquement conforme, Thèse de Docteur-Ingénieur, Toulouse (1976). 